\title{
Chatter identification in milling of Inconel 625 based on recurrence plot technique and Hilbert vibration decomposition
}

\author{
Pawet Lajmert ${ }^{1, *}$, Rafał Rusinek ${ }^{2}$, and Bogdan Kruszyński ${ }^{1}$ \\ ${ }^{1}$ Lodz University of Technology, Institute of Machine Tools and Production Engineering, Stefanowskiego 1/15 Street, Lodz, Poland \\ ${ }^{2}$ Lublin University of Technology, Department of Applied Mechanics, Nadbystrzycka 36 Street, Lublin, Poland
}

\begin{abstract}
In the paper a cutting stability in the milling process of nickel based alloy Inconel 625 is analysed. This problem is often considered theoretically, but the theoretical finding do not always agree with experimental results. For this reason, the paper presents different methods for instability identification during real machining process. A stability lobe diagram is created based on data obtained in impact test of an end mill. Next, the cutting tests were conducted in which the axial cutting depth of cut was gradually increased in order to find a stability limit. Finally, based on the cutting force measurements the stability estimation problem is investigated using the recurrence plot technique and Hilbert vibration decomposition method.
\end{abstract}

\section{Introduction}

The need to produce responsible machine parts in aerospace, chemical process or seawater applications requires the use of special metal alloys, such as nickelchromium-molybdenum alloys, e.g. Inconel 625. Inconel 625 belongs to category of superalloys, which have high tensile strength in high temperatures, good fatigue strength and outstanding corrosion and oxidation resistance [1]. The properties of this material that make it an excellent choice in many demanding applications causes however, many difficulties in its machining. Additionally, in industry, because of competitiveness reasons, there is a constant need for growth in productivity and product quality that requires the material removal rate to be increased [2]. A higher productivity may be achieved using high speed machining (HSM) which however can induce so called self-excited vibrations caused by a regenerative mechanism [3, 4]. To avoid regenerative chatter, the working region corresponding to stable machining must be identified. For this purpose, a stability lobe diagrams (SLDs) are usually created, where rotational speed and depth of cut determine the boundary between stable and unstable cutting [3]. However, due to non-stationary nature of cutting processes the exact boundary position is difficult to predict in advance and may change for different technological cases. This in turn forces the machine tool operators to use undervalued cutting parameters. Although, there exist methods for eliminating self-excited vibrations they are quite difficult to apply in industrial practice. From these reasons there is a need to use automatic supervision systems which could diagnose the process online and change the cutting parameters when some symptoms of process instabilities appear [5-9]. Moreover, some papers focus on different methods of chatter identification directly from workpiece displacement or cutting force signals [3, 10-14].

This paper deals with the problem of stability limit prediction of chatter vibrations in milling process of Inconel 625 on the basis of experimental measurements of cutting force components. In order to find the initial point of chatter vibrations, the recurrence quantification analysis and Hilbert vibration decomposition are employed $[15,16]$.

\section{Experimental tests}

The experimental tests on Inconel 625 were performed using the Haas MiniMill CNC milling machine. The experimental setup, presented schematically in Fig. 1, is composed of two parts: a modal analysis system and a dynamometer for measuring cutting forces. The former was used to measure viscoelastic properties of the machine-tool system. The modal system consists of the PCB-086C03 modal hammer, the PCB-352B10 accelerometer and the NI-9234 data acquisition card. The rotating, piezoelectric dynamometer Kistler 9123C can measure three components $F_{x}, F_{y}$ and $F_{z}$ of the resultant cutting force and torque $M_{z}$. The dynamometer is connected to the Kistler 5223 signal conditioner and the 2855A4 data acquisition card. Both measurement systems are integrated with a PC computer and controlled by the DynoWare software.

The measurements were carried out in two steps. First, a single point impact test is performed to determine modal parameters of the cutting system, that is: stiffness, natural vibration frequency and a damping ratio. The modal hammer is used to excite the tool, and then the output signal is measured by the low mass accelerometer

\footnotetext{
* Corresponding author: pawel.lajmert@p.lodz.pl
} 
mounted at the tool tip. The test is repeated for the two perpendicular directions $x$ and $y$.

Next, the modal parameters are implemented to the CutPro9 software as a frequency response function (FRF) in order to determine stability lobes diagram (SLD). The SLD, generated for the up-milling of Inconel 625 using a $12 \mathrm{~mm}$ diameter end mill cutter with four flutes is shown in Fig. 2. The radial depth of cut, $a_{e}$, is set to $4 \mathrm{~mm}$ and the feed per tooth, $f_{z}$, is set to $0.05 \mathrm{~mm}$. Stable cutting occurs in the region below the stability lobes, while unstable machining should occur above the lobes. The unconditionally stable region is below the critical cutting depth $a_{p c r}=0.65 \mathrm{~mm}$. Then, the cutting process is stable regardless the spindle speed.

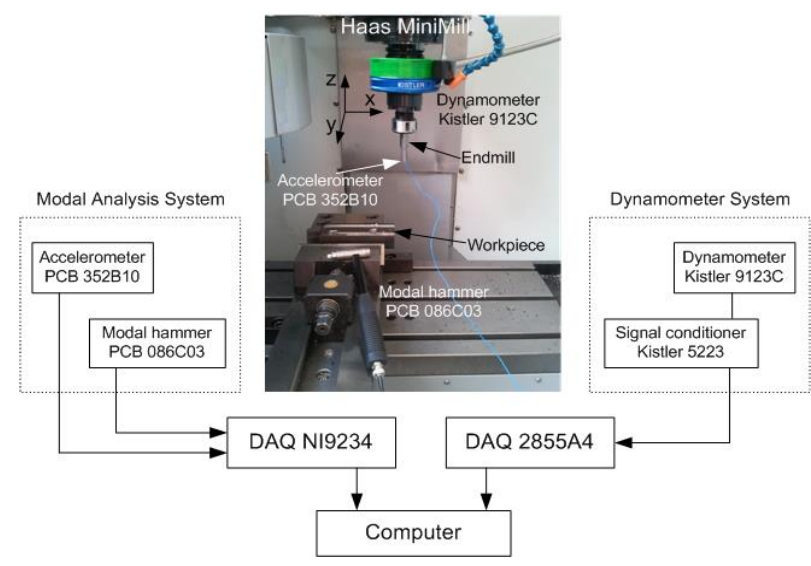

Fig. 1. Experimental setup scheme.

The second stage of experiment is performed on the milling machine in order to verify the SLD and to explain chatter vibrations nature. The cutting parameters are set as follows: spindle rotational speed $n=1900 \mathrm{rpm}$, radial depth of cut $a_{e}=4 \mathrm{~mm}$, feed per tooth $f_{z}=0.05 \mathrm{~mm}$, while the axial depth of cut $a_{p}$ is being increased from 0 to $1 \mathrm{~mm}$ during the test. The cutting force components, $F_{x}, F_{z}$, and the torque, $M_{z}$, are measured and recorded with a sampling rate of $2 \mathrm{kHz}$.

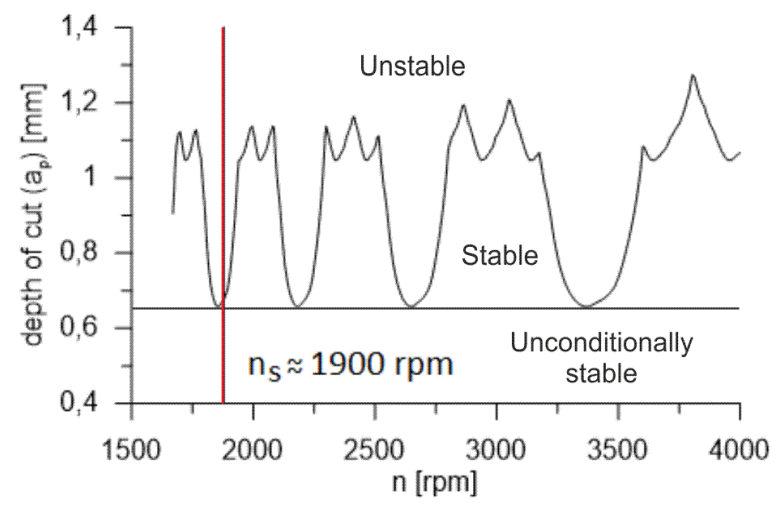

Fig. 2. Stability lobes diagram for Inconel 625 milling.

The analyses in the following section are undertaken to verify whether the critical depth of cut, presented in Fig.2, is found properly as well as to identify chatter vibrations component in the measured signal.

\section{Time series analysis}

In this section the analysis of the force time series is done for the application in stability and chatter identification of milling process. To achieve this aim, the recurrence plot (RP) technique and Hilbert vibration decomposition (HVD) methods are applied.

\subsection{Recurrence plot technique}

Based on the experiment and CutPro analysis the SLD is achieved. However, industry practice suggests that the stability boundary is not quite proper. Therefore, the obtained stability limit from the SLD is verified here experimentally using the recurrence plot (RP) technique. The RP approach provides a qualitative interpretation of hidden patterns of dynamical systems based on phase space reconstruction. This technique was originally introduced by Eckmann [15]. According to the RP theory any time series can be presented as a delayed vector in an $m$-dimensional space called "the reconstructed phase space". The embedding parameters, i.e. a dimension $m$ and a time delay $d$ are estimated by applying the average mutual information function (AMI) and the false nearest neighbours method (FNN). More information about the AMI and FNN functions can be found in the papers $[3,10,17]$.

In this study, we applied the Tisean software [18] to determine the embedding parameters. A recurrence plot is a graph which shows all time instants at which a state of a dynamical system recurs. If the trajectory in the reconstructed phase space returns at time $i$ into the neighbourhood of $\varepsilon$ where it was before at time $j$, then the results are plotted as black point in RP. Black points create a specific pattern which can be analysed to achieve some information about system dynamics. From a practical point of view, RPs can be presented also by means of the recurrence quantification analysis (RQA) which is not considered in this contribution. The RP for the cutting force components $F_{x}$ and $F_{z}$ are presented in Fig. 3 and 4 . Note that during the milling test depth of cut $a_{p}$ was being constantly increasing from 0 to $1 \mathrm{~mm}$.

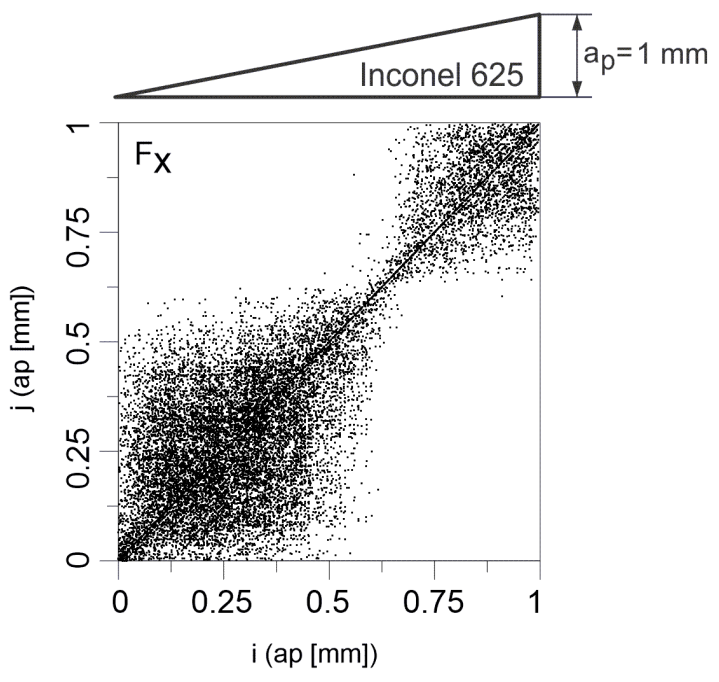

Fig. 3. Recurrence plots for cutting forces $F_{x}$. 


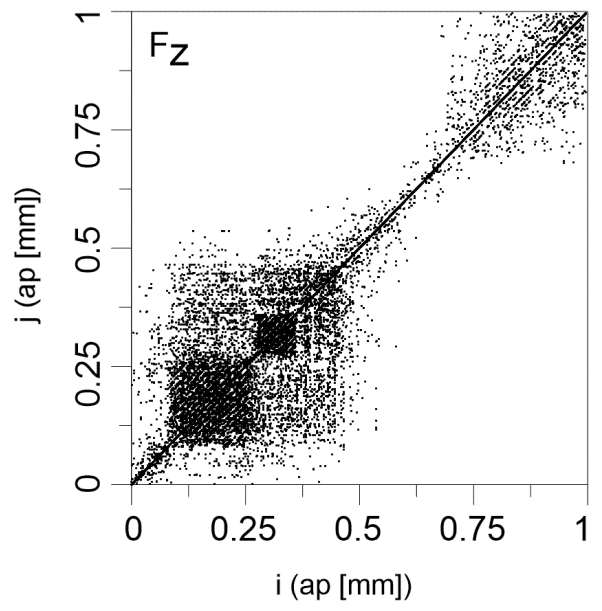

Fig. 4. Recurrence plots for cutting forces $F_{z}$.

The transition from chatter free milling to chatter is visible at $a_{p}=0.5 \mathrm{~mm}$, that is just before the value obtained from CutPro. In case of transition from chatter free to chatter region the change of RP pattern is observed both for $F_{x}$ and $F_{z}$ force.

\subsection{Hilbert vibration decomposition}

The Hilbert vibration decomposition was developed by Feldman for separation of non-stationary and multicomponent dynamical signals [16, 19]. In contrast to Fourier transform, in Hilbert transform it is assumed that the signal may be represented by a single, but amplitude and frequency modulated sinusoidal waveform [19]. As a consequence, the HVD method, which is based on Hilbert transform, allows to decompose the original signal into a sum of quasiharmonics with slow-varying instantaneous amplitudes and frequencies:

$$
x(t)=\sum_{i=1}^{N} A_{i}(t) \cos \left(\int_{0}^{t} \omega_{i}(t) d t\right)
$$

where $A_{i}(t)$ and $\omega_{i}(t)$ are the instantaneous amplitude and frequency of the $i$-th component. The decomposition of the initial signal $x(t)$ starts with the estimation of its instantaneous frequency $\omega(t)$ with the use of Hilbert transform. For nonstationary signals with multiple components the instantaneous frequency $\omega(t)$ is composed of two parts. The first is slow varying frequency of the largest energy component and the other is a rapid varying part which oscillate around the first one [16]. Thanks to this property, the mean value of instantaneous frequency $\omega(t)$ is equal to the instantaneous frequency $\omega_{l}(t)$ of the largest energy component. Having the instantaneous frequency $\omega_{l}(t)$ it is possible to extract from the signal its instantaneous amplitude $A_{l}(t)$ using a synchronous demodulation algorithm [16, 19]. Finally, based on the instantaneous frequency and amplitude, the first isolated component $x_{I}(t)$ with the highest energy is generated. The isolated component is subtracted from the original signal and the whole procedure is repeated to isolate the subsequent components. The flowchart of the HVD method is shown in Fig. 5.

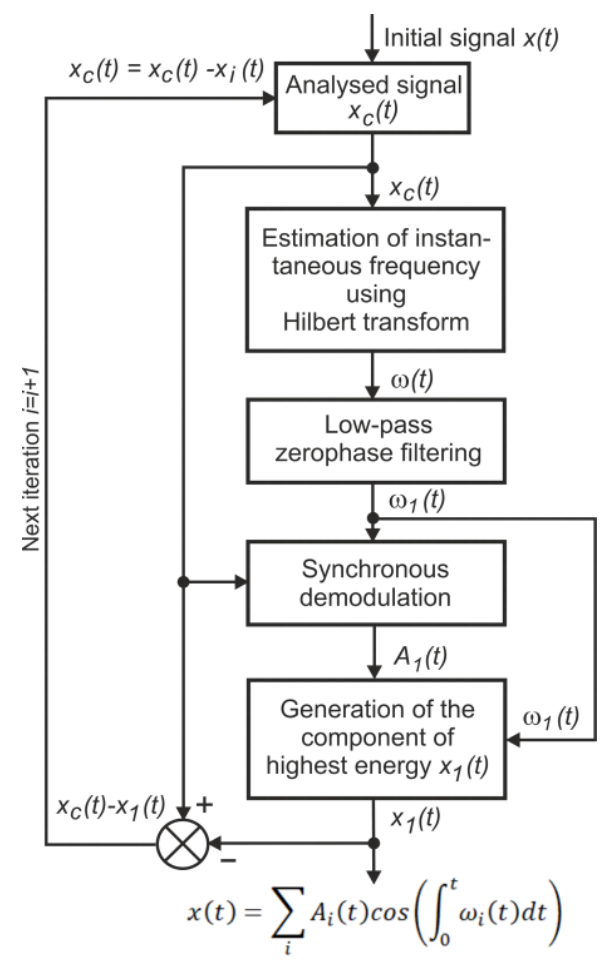

Fig. 5. Flowchart of HVD method.

The presented method was used to extract components from real force measurements. Based on a preliminary experiments the number of desired components to be isolated was established. In Fig. 6 the first three dominant components, isolated from $F_{z}$ force time series are shown.
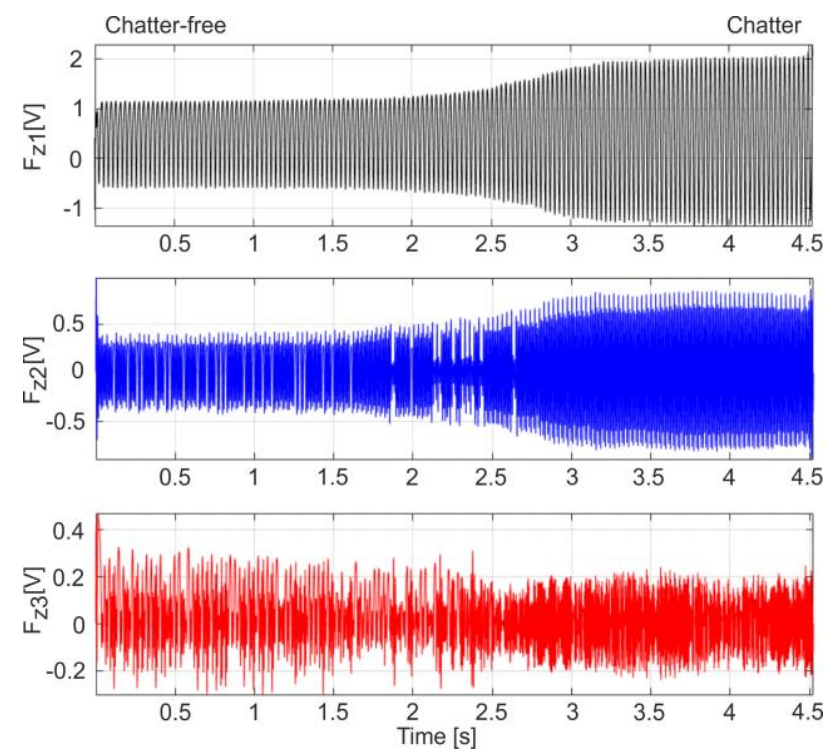

Fig. 6. Decomposed components of force $F_{z}$ time series.

The first component is responsible for the force changes caused by the material removal by the individual wings of the end mill cutter (tool rotational speed). The second 
component may be responsible for the vibrations of the machine tool structure, while the third one corresponds to a milling cutter vibrations. In Fig. 7 the course of this component (red line) for chatter-free and chatter milling is presented. It may be seen that for stable machining, the milling cutter vibrations are dampened after each cutter engagement. On the other hand for chatter milling the cutter vibrations are not dampened.

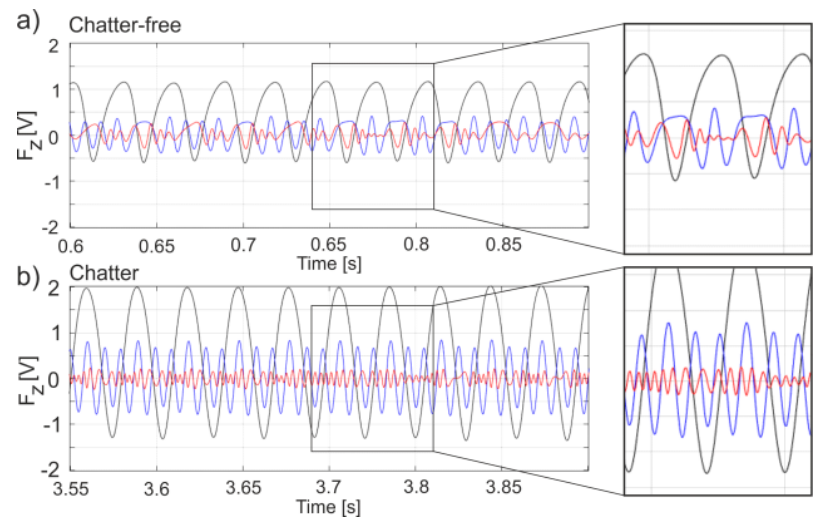

Fig. 7. The course of the three components for: a) chatter-free and b) chatter milling.

In Fig. 8 changes of instantaneous amplitudes for the three extracted components are presented. Particularly for the first and second component (black and blue line) a growth of the amplitude may be noticed, being the evidence of chatter vibrations.

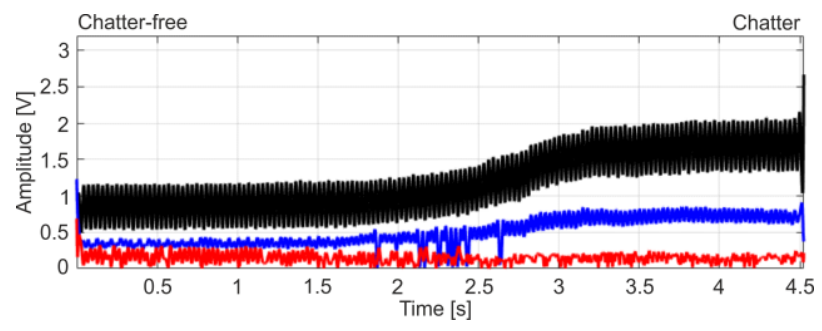

Fig. 8. Changes of the instantaneous amplitude for the three decomposed signal components.

In Fig. 9 Hilbert spectrum for three decomposed components are presented. As may be seen the increase of variance of instantaneous frequency for third component (red one) is clearly visible.

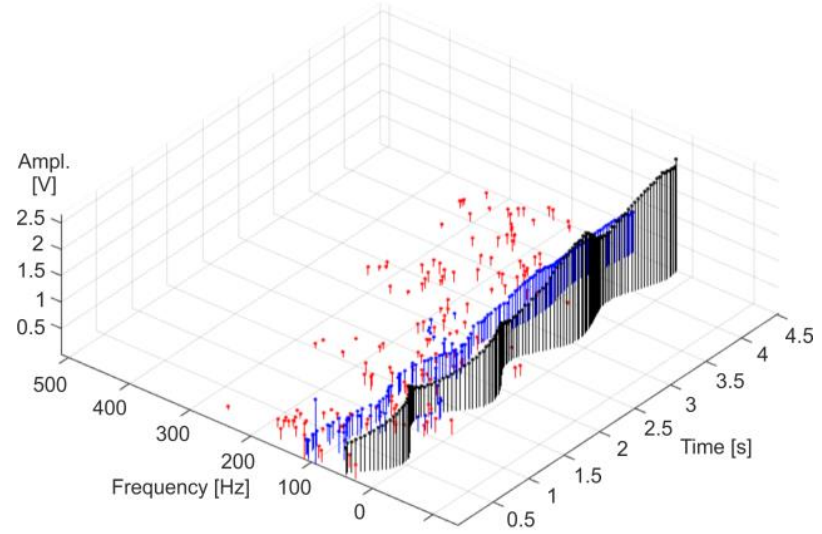

Fig. 9. Hilbert spectrum for the three decomposed components.

\section{Conclusions}

The paper focused on assessment of milling process stability by the recurrence plot technique and Hilbert vibration decomposition. The recurrence plots drawn for the milling force components show exactly the moment when chatter vibrations appear. The critical depth of cut, determined by means of RP is a bit different compared to the one obtained by means of modal test and CutPro software. It was shown that dynamical features of individual force components can be revealed by means of advanced signal analysis methods, e.g. the HVD method. The use of this method enables determination of the force signal components which are related to individual cutting processes or machine tool vibration modes. Therefore, these components have a greater potential to distinguish between chatter-free and chatter states. It must be noted that major disadvantage of HVD method is the difficulty in proper selection of algorithm parameters, mainly the parameters of low-pass filter.

\section{References}

1. E. O. Ezugwu, Z. M. Wang and A. R. Machado, J. of Materials Processing Technology 86 (1999) 1-16.

2. Gdula M., Burek J. Zylka L., Plodzen M., Aircraft Enginering and Aerospace Technology, (2015).

3. Kecik K., Rusinek R., Warminski J., Int. J. of Bifurcation and Chaos 21/10, 2943-2954 (2011).

4. R. Rusinek, Maintenance and Reliability 3, 48-55 (2010).

5. B. Kruszynski, P. Lajmert, Proc. Inst. Mech. Eng., Part B: J. Eng. Manuf. 220, 355-363 (2006).

6. B. Krusznski, P. Lajmert, CIRP Ann. - Manuf. Technol. 54/1, 305-308 (2005).

7. P. Lajmert, M. Sikora, B. Kruszynski, D. Ostrowski, Lecture Notes in Mech. Eng, Adv. in Manufacturing, 707-716 (2018).

8. Zylka L., Burek J., Mazur D., Adv. in Production Engineerign \& Management 12, 221-232 (2017).

9. Wolszczak P., Lygas K., Litak G., Adv. In Science and Technology Research Journal 11/1, 96-103 (2017).

10. Rusinek R., J. of Engineering Manufacture, 226/12, 1976-1985 (2012).

11. Litak G., Kecik K., Rusinek R., Latin Americal J. of Solids and Structures 10/1, 133-138 (2013).

12. Litak G., Rusinek R., Kecik K., Rysak A., Syta A., Discontinuity and complexity in nonlinear physical systems, Springer 359-367 (2014).

13. R. Rusinek, P. Lajmert, K. Kęcik, B. Kruszynski, J. Warmiński, Int. J. of Mechanical Sciences 99, 196207 (2015).

14. Kecik K., Borowiec M., Rusinek R., European Physical Journal Plus 131/1, (2016).

15. J.-P. Eckmann, S.O. Kamphorst, D. Ruelle, Europhys. Lett. 4, 973-977 (1987). 
16. M. Feldman, J. of Sound and Vibration 295, 518530 (2006).

17. R. Rusinek, Int, J. of Non-Linear Mechanics 45, 458-462 (2010).

18. R. Hegger, H. Kantz, T. Schreiber, The TISEAN package, Chaos 9, 413-435 (1999).

19. M. Feldman, Mechanical Systems and Signal Processing 25, 735-802 (2011). 